\title{
O Transtorno do Déficit de Atenção e Hiperatividade
}

Catarina Fernandes Macêdo Bezerra ${ }^{1}$; Maria Valéria Leimig Telles ${ }^{2}$; Maria Iaponira Fernandes Macêdo Bezerra ${ }^{3}$; Samuel de Oliveira Ribeiro ${ }^{I}$; Noyla Denise Alencar de Sál

Resumo: O transtorno do déficit de atenção e/ou hiperatividade (TDAH) é uma doença de alta prevalência em crianças em idade escolar. Erroneamente entendido anteriormente como um diagnóstico de baixa morbidade, o TDAH é reconhecido atualmente como uma condição importante, não só pelo forte impacto funcional e social como também pela alta prevalência de comorbidades psiquiátricas. O TDAH é um distúrbio neurofisiológico, com sinais de falta de atenção e impulsividade não adequadas ao nível de desenvolvimento, prejudiciais a aprendizagem e com impacto na qualidade de vida dessas crianças. Não existe marcador biológico para o TDAH, seu diagnóstico é clínico, baseado em entrevista com o paciente, pais, professores e pessoas que lidam com o portador. O objetivo visa ressaltar os aspectos mais importantes da avaliação do TDAH, consultando a bibliografia mais pertinente para avaliação deste transtorno. O presente estudo busca uma revisão atual dos principais aspectos do histórico, epidemiologia, neurobiologia, quadro clínico, comorbidades, diagnóstico, evolução e tratamento do transtorno.

Palavras-chave: Transtorno de Déficit de Atenção/Hiperatividade - TDAH. Criança. Adolescente.

\section{Attention Deficit Hyperactivity Disorder}

\begin{abstract}
Attention deficit/hyperactivity disorder (ADHD) is a highly prevalent disorder among school age children. Once understood as a common and mild disorder restricted to childhood, ADHD is now recognized as an important condition because of its poor outcome and strong association with comorbidities. ADHD is a neuropsychological disorder with signs of inattention and impulsivity is not appropriate to the level of development, harmful to learning and impact on quality of life of these children. There is no biological marker for ADHD, its diagnosis is clinical, based on interviews with patients, parents, teachers and people who deal with the carrier. The paper aims at pointing out the most important aspects of ADHD evaluation through the most pertinent bibliography for assessment of this disorder. This study aims to present a review of the main aspects of the history, epidemiology, neurobiology, clinical features, co morbidities, diagnosis, progression and treatment of the disorder.
\end{abstract}

Keywords: Disorder Attention Deficit Disorder - ADHD. Child. Adolescent.

\section{Introdução}

O Transtorno de déficit de atenção e hiperatividade (TDAH) é um comprometimento neurocomportamental caracterizado por desatenção e hiperatividade/ impulsividade que, frequentemente, resulta em um prejuízo funcional substancial (VISSER; LESENE, 2003 apud ABREU; OLIVEIRO, 2010).

\footnotetext{
${ }^{1}$ Médico(a) pela Faculdade de Medicina Estácio - Juazeiro do Norte - CE.

${ }^{2}$ Médica, Doutorado em Neurologia/Neurociências pela Universidade Federal de São Paulo - UNIFESP. Professora da Faculdade de Medicina Estácio em juazeiro do Norte - CE.

${ }^{3}$ Farmacêutica pela Universidade Federal do Ceará.
} 
O TDAH vem sendo considerado um dos distúrbios comportamentais mais frequentes na infância, com taxas de prevalência entre 3\% e 7\% em crianças em idade escolar, segundo o Manual diagnóstico e estatístico de transtornos mentais DSM-IV (AMERICAN PSYCHIATRIC ASSOCIATION, 2002 apud ASSEF; CAPOVILLA; CAPOVILLA).

Muitos fatores etiológicos foram propostos para esse transtorno e cada um deles é capaz de levar à mesma apresentação comportamental. Os principais fatores implicados na etiologia do TDAH são de natureza genética, biológica e psicossocial. Não existem testes laboratoriais, achados de neuroimagem ou perfis em testes neuropsicológicos que sejam patognomônicos de TDAH. Assim, o diagnóstico de TDAH é essencialmente clínico, baseado em critérios claros e bem definidos (FONTANA et al., 2007).

Com base nos sintomas, os indivíduos portadores de TDAH podem ser classificados em três subtipos: misto, predominantemente hiperativo e predominantemente desatento. Existem diferenças importantes entre os subtipos que incluem os sintomas centrais de desatenção, hiperatividade e impulsividade, mas não se limitam a eles (FONTANA et al., 2007).

Em meninos a incidência é maior, aproximadamente nove meninos para um caso de meninas, estas podendo demonstrar o tipo predominantemente desatento com mais frequência (PETRIBÚ; VALENÇA; OLIVEIRA, 1999 apud LOPES; NASCIMENTO; BANDEIRA, 1999).

O TDAH causa grande impacto na vida de seus portadores. Estes apresentam relações interpessoais instáveis e tumultuadas, baixo desempenho acadêmico e profissional, o que acaba por acarretar enormes prejuízos no funcionamento familiar e social. Este impacto é ainda amplificado pelas altas taxas de comorbidades com outras doenças psiquiátricas, pois sabe-se que $77 \%$ dos adultos com TDAH apresentam outro transtorno psiquiátrico concomitante. Os transtornos comórbidos mais comuns são: Depressão Maior, Transtornos de Ansiedade, Transtornos de Humor Bipolar, Transtornos de Abuso de Substâncias, Transtornos de Personalidade e Alterações de Conduta na Idade Adulta (GREVET; ABREU; SHANSIS, 2003).

Estudos longitudinais demonstraram que o transtorno de déficit de atenção/hiperatividade (TDAH) persiste na vida adulta em torno de 60\% a 70\% dos casos (BARKLEY et al., 2002 apud MATTOS; COUTINHO, 2007), sendo as diferenças encontradas nas taxas de remissão provavelmente secundárias às diferentes definições de TDAH ao longo do tempo e aos critérios para o seu diagnóstico (MATTOS; COUTINHO, 2007 apud BIEDERMAN et al., 2000). O diagnóstico de TDAH em adultos é realizado utilizando-se critérios bem definidos, como os empregados pelo sistema DSM. Os sintomas listados no DSM-IV para o diagnóstico de crianças e adolescentes foram adaptados para adultos na escala auto-aplicada ASRS - Escala de Auto-Relato do TDAH em Adultos (Adult Self- 
Report Scale) (KESSLER et al., 2005 apud MATTOS; COUTINHO, 2007) validada recentemente no nosso meio (MATTOS et al., 2006a).

O tratamento farmacológico clássico baseia-se na utilização de psicoestimulantes para os pacientes com TDAH, sejam eles crianças, adolescentes ou adultos. Não é mais admissível, nos dias atuais, que um paciente com TDAH não receba medicação, uma vez que se sabe que a dimensão da desatenção responde muito pouco, ou quase nada, às abordagens psicoterápicas. Contudo, os tratamentos psicoterápicos possuem um papel fundamental na recuperação do paciente (GREVET; ABREU; SHANSIS, 2003).

O presente trabalho apresentará elementos teóricos pertinentes ao TDAH, que é caracterizado como uma patologia de alta prevalência na infância, sobretudo em idade escolar e com impacto importante na qualidade de vida dessas crianças. O estudo visa promover novos conhecimentos sobre o ambiente em que vivem as crianças com esse transtorno e o entendimento sobre recentes informações acerca do diagnóstico, manejo e tratamento, ampliando sua compreensão.

Nesta perspectiva, o objetivo do presente estudo foi realizar uma revisão da literatura a respeito do assunto (TDAH), atualizada e de fácil compreensão para os profissionais de saúde, pais, profissionais de educação e pacientes.

\section{Método}

A revisão de literatura refere-se à fundamentação teórica adotada para tratar o tema ou problema da pesquisa, por meio da análise da literatura publicada e traçar um quadro teórico, bem como uma estruturação conceitual que dará sustentação ao desenvolvimento da pesquisa (SILVA, 2001).

De acordo com Luna (1997), um dos objetivos de uma revisão de literatura é recuperar a evolução de um conceito, tema, abordagem ou outros aspectos fazendo a inserção dessa evolução dentro de um quadro teórico de referência que explique os fatores determinantes e as implicações das mudanças.

Para Lakatos e Marconi (1991), a revisão de literatura apresenta várias contribuições, pois acessa informações atualizadas sobre o tema ou problema pesquisado, levanta dados já abordados em publicações antigas e dá oportunidade de comparação de pontos de vista a respeito do tema de diversos autores.

O estudo foi realizado com início em dezembro de 2013 e concluido em abril de 2014. Foram utilizados conteúdos de sites de coleção de artigos científicos como PUBMED, SCIELO e LILACS, além de livros nacionais. 
Artigos científicos e livro, em língua portuguesa e inglesa, dos últimos anos (1990-2013), que contenham informações pertinentes aos objetivos desta pesquisa. Estudos exploratórios, como revisão de literatura, pesquisa documental e estudos descritivos.

\section{Revisão da Literatura}

\section{Definição e Histórico}

O transtorno de déficit de atenção e hiperatividade é uma síndrome com alta prevalência em crianças e adolescentes, que causa discrepância referente ao comportamento esperado para a faixa etária e inteligência. Acarreta prejuízo para o desenvolvimento dos indivíduos acometidos em diferentes domínios da integração social (PEREIRA; ARAÚJO; MATTOS, 2005).

Esta alteração se chama Transtorno de déficit de atenção e hiperatividade/impulsividade, abreviado como TDAH em português ou ADHD em inglês. Ele já foi chamado de "ADD" ou "DDA" em português, para se referir aos transtornos que não possuem sintomas de hiperatividade, mas essas nomenclaturas já foram abandonadas (MATTOS, 2013b).

O TDAH é uma das principais causas de procura de ambulatório de saúde mental de crianças e adolescentes (FARAONE et al., 2003; ROHDE et al., 2000). As características nucleares do transtorno na infância são a desatenção, a hiperatividade e a impulsividade. Elas afetam de várias formas o desempenho acadêmico, os relacionamentos familiares e sociais e o ajustamento psicossocial, portanto, devem ser alvo de intervenções (ROHDE et al., 2004).

Esses pacientes em muitos casos são inquietos, porém nem sempre, não permanecendo parados, nem sossegados por muito tempo e detestam coisas monótonas e repetitivas, além de serem impulsivas no seu dia a dia. São pessoas que vivem trocando de interesses e planos e têm dificuldades em levar as coisas até o final. Apesar desses sintomas serem muito frequentes e causarem vários problemas na vida do indivíduo, podem ficar muito tempo ou até mesmo a vida inteira sem serem considerados como expressão de um transtorno, que não é diagnosticado e nem tratado de maneira correta (MATTOS, 2013b).

Para Bauermeister (2008) apud Bauermeister (2009), o TDAH caracteriza-se pelas dificuldades para responder adequadamente em situações que exige manter a atenção e inibir ou regular a conduta, podendo trazer sofrimento às pessoas com essas manifestações de comportamento e afetar sua capacidade para funcionar adequadamente. Quando as características predominantes são a desatenção e a hiperatividade-impulsividade, o padrão de comportamento é chamado de transtorno de 
déficit de atenção com hiperatividade-impulsividade, tipo combinado. Quando a característica predominante é a hiperatividade-impulsividade, o padrão de conduta se denomina transtorno do déficit de atenção com hiperatividade-impulsividade, tipo predominantemente hiperativo-impulsivo. Quando a característica é a falta de atenção, o padrão de comportamento é denominado transtorno por déficit de atenção com hiperatividade-impulsividade, tipo predominantemente desatento.

As primeiras referências aos transtornos hipercinéticos ou transtorno de déficit de atenção e hiperatividade e/ou impulsividade na literatura médica surgiram na metade do século XIX. Foi em 1865, na poesia do médico alemão Heinrich Hoffman, que escrevia poemas sobre muitas das doenças infantis que encontrou em sua prática médica. Contudo o crédito científico costuma ficar com George Still e Alfred Tredgold, que foram os pioneiros a dedicar atenção clínica séria a uma condição comportamental infantil que se aproximava do que hoje se conhece como TDAH (BARKLEY, 2008).

O primeiro a descrever o TDAH, em 1902, foi o médico pediatra, George Still que publicou o trabalho no jornal médico (Lancet) (ROHDE; HALPERN, 2004). Ele analisou os sintomas em um grupo de crianças, no qual observou a existência de um "defeito na conduta moral". Essa alteração resultava em uma inabilidade da criança para seguir limites, gerando sintomas de inquietação, desatenção e impaciência. Assim, Still formulou a hipótese de que esta condição não se tratava de uma má-criação ou perversidade da criança e sim a de uma herança biológica ou a um problema no parto (BENCZIK, 2002 apud LIMA, 2011).

Em 1937, Charles Bradley mostrou uma linha de relação da hiperatividade com o biológico, diante da descoberta acidental de que alguns estimulantes do sistema nervoso central ajudavam crianças hiperativas a se concentrarem melhor, a aprenderem melhor e a ficarem mais calmas. Esta descoberta foi contrária aos pensamentos da época, pois perceberam que os medicamentos estimulantes em pessoas que não apresentavam esse quadro, produziam efeito inverso, ou seja, produziam um aumento de atividade no sistema nervoso central (BENCZIK, 2002 apud LIMA, 2011).

Sua nomenclatura vinha sofrendo várias alterações. Nos anos 40, surgiu a designação de "lesão cerebral mínima" que em 1962 foi modificada para "disfunção cerebral mínima", reconhecendo-se que as alterações características da síndrome está mais relacionada com disfunções em via nervosa do que a lesões. O CID-10 e DSM IV, que são sistemas classificatórios utilizados em psiquiatria, apresentam mais similaridade do que diferenças nas diretrizes de diagnóstico para o transtorno, embora utilizem nomenclaturas diferentes, transtorno de déficit de atenção e hiperatividade no DSM-IV e transtorno hipercinético no CID-10 (ROHDE et al., 2000).

O primeiro DSM surgiu em 1952 e tinha como bases o pensamento de Adolf Meyer. O DSM II, publicado em 1968, foi reestruturado com base na linguagem psicanalítica. Para Young (1995) apud Caliman (2009), publicação do DSM III, em 1980, materializou definitivamente o estilo de pensamentos psiquiátricos. O DSM III mudou drasticamente o sistema americano de classificações das 
doenças mentais. Os números de diagnósticos foram multiplicados, as referências a autores e teorias banidas. Segundo Blashfield (1998) apud Caliman (2009), o manual tornava-se o emblema da autoridade e neutralidade científica. Foi no contexto de elaboração do DSM III e das transformações, na história da psiquiatria, que o diagnóstico da desordem do déficit de atenção (DDA) surgiu como uma categoria psiquiátrica. $\mathrm{O}$ antigo diagnóstico era confuso e problemático do transtorno de hiperatividade era definido como uma desordem da atenção (CALIMAN, 2009).

Nas décadas de 60 e 70, a patologia da hiperatividade foi analisada e estudada e estava no centro do debate educacional e médico, mas para a psiquiatria ela trazia problemas. Seu diagnóstico era impreciso e subjetivo. Era preciso construir uma nova categoria diagnóstica, mais unificada, objetiva e clara. A partir dos anos 70, vários pesquisadores começaram a investigar a hiperatividade e o problema de desatenção através do uso de medidas psicofisiológicas. Um grupo da Universidade de McGill exerceu um papel importante na constituição de um novo olhar molecular e neurofisiológico que redefinia a forma de diagnosticar e tratar os considerados déficits atentivos e as manifestações da hiperatividade infantil. A década de 80 trouxe novas perspectivas e promessas para a psiquiatria, principalmente no cenário americano. Nestes muitos fatores de acontecimentos, o diagnóstico do Transtorno de déficit de atenção e hiperatividade teve sua aparição oficial ao ser incluído no DSM IV, em 1994 (CALIMAN, 2009).

Foi a partir dessa década que o Transtorno de déficit de atenção e hiperatividade (TDAH) vem tomando lugar de importância na vida dos pais e profissionais de saúde mental, sendo considerado o principal distúrbio psicológico em crianças e adolescentes. Com o crescimento de grupos de apoio e com um maior acesso a informações, mais crianças estão sendo diagnosticadas e tratadas corretamente (CARVALHO et al., 2012).

Um artigo publicado pela Heritage Foundation (1999) apud Caliman (2008), descrevia o “cenário TDAH" nos anos 90. De acordo com a autora, desde o final dos anos 80 e inicio dos anos 90, o mundo presenciou uma explosão de informações sobre TDAH e a ritalina, sendo na época o medicamento mais utilizado no tratamento do transtorno. Vários livros sobre o assunto alcançaram a categoria de bestseller. Nessa época o adulto TDAH tornava-se parte do público interessado (CALIMAN, 2008).

O TDAH até o momento considerado um diagnóstico exclusivamente infantil, começava a ser visto como uma patologia do desenvolvimento que continuava na vida adulta, um quadro crônico incurável. Principalmente nos Estados Unidos da América, a compreensão e tratamento dessa desordem tornavam-se uma necessidade vinculada à gestão social e econômica da nação. Em 2004 foi reconhecido pela Resolução 370 como um dos problemas mais graves e importantes de saúde publica americana. O TDAH abrangia de 3 a 7\% das crianças e adolescentes americanos em idade escolar e 
4\% dos adultos. Devido essa Resolução, teve sua entrada nas datas oficiais do país com a proclamação do dia 7 de setembro como o "Dia da Consciência Nacional sobre o TDAH" (CALIMAN, 2008).

De acordo com Pretry et al. (2011) apud Lima (2011), historicamente é possível perceber que cada período parece trazer a tona alguma doença ou perturbação que se torna a principal preocupação de determinada população e, também passa a ser a mais percebida, mesmo onde não existe. Passa a causar temor às pessoas, que é o caso da TDAH(SILVA, 2003 apud LIMA, 2011). É preciso esclarecer que as crianças com TDAH não são maldosas, malcriadas ou mal educadas pelos pais. Elas são injustamente acusadas, quando na verdade possuem um transtorno que as fazem agir de maneira impulsiva, desatenta e agitada (LIMA, 2011).

\section{Epidemiologia}

A prevalência do transtorno tem sido pesquisada em inúmeros países. Diferenças encontradas nas taxas de prevalência refletem mais diferenças metodológicas, como do tipo da amostra, delineamento, fonte de informação, idade, critérios diagnósticos e a forma como são aplicados, do que reais diferenças culturais na construção do diagnóstico e do transtorno. Estudos nacionais e internacionais tendem a encontrar prevalências em torno de 3-6\% em crianças em idade escolar (NAGEL et al., 2011; ROHDE; HALPERN, 2004).

É uma condição cuja prevalência foi documentada em muitas partes do mundo, como a Alemanha, Porto Rico e Taiwan. Pesquisas recentes realizadas pelo National Comorbidity Survey realizada em aproximadamente 10.000 pessoas nos Estados Unidos mostraram uma taxa da prevalência TDAH em adulto de $4 \%$ (HECHTMAN, 2005).

Estudos têm demonstrado que o TDAH aparece na primeira infância, por volta dos 3 anos de idade, com maior frequência no sexo masculino do que no sexo feminino, em uma proporção de 4 para 1. Na população com idade entre 6-12 anos a prevalência é em torno de 4 a 12\% (POLÔNIO, 2009 apud CARVALHO et al., 2012).

No Brasil, e em outras áreas geográficas tem a prevalência estimada em torno de 3,5 a 8\%. Sendo mais freqüente em meio urbano em relação ao meio rural e aumenta em função de condições sócio-econômicas mais precárias (POSSA; SPANEMBERG; GUARDIOLA, 2005).

Segundo MATTOS (2013b) acreditava-se que o TDAH era mais frequente em meninos do que meninas. Mas essa afirmação parecia ser verdadeira no ambiente clínico e de tratamento. Quando foram realizadas pesquisas na população geral, como em uma escola, por exemplo, parece existir uma igualdade estatística no número de meninos e meninas com TDAH. 
Na fase adulta há tanto pacientes homens como mulheres em ambientes clínicos ou na população em geral (MATTOS, 2013b). Uma pesquisa realizada por Fontana et al. (2007), em 4 escolas públicas do município de São Gonçalo (estado do Rio de Janeiro), com 461 escolares de $1^{\mathrm{a}}$ à $4^{\mathrm{a}}$ series do ensino fundamental com idade entre 6 e 12 anos, visualizou a prevalência de TDAH, considerando o conjunto das 4 escolas, de 13\%. A proporção do sexo masculino: sexo feminino foi 2:1 e o subtipo de mais frequente foi o misto com $61,7 \%$ dos casos.

Em mais de 50\% dos casos de TDAH existe associação com outras condições clínicas, como transtornos de aprendizado, transtorno de humor e da personalidade, transtorno de ansiedade, transtorno disruptivos do comportamento e uso de substâncias psicoativas e de álcool (ROHDE et al., 2000; VIDAL-ESTRADA et al., 2012).

Essa associação frequente de TDAH com outros transtornos é avaliada por diversos profissionais da área da saúde, como fonoaudiólogos, psicólogos e terapeutas ocupacionais. Dessa forma, é proposta a necessidade da abordagem multidisciplinar na avaliação, diagnóstico e intervenção para estas crianças (PEREIRA; ARAÚJO; MATTOS, 2005).

Essas crianças encontram dificuldades no estabelecimento das relações. Por não conseguirem estabelecer bons contatos sociais, poderão ser excluídas dos grupos a que pertencem. No ambiente escolar, fica evidente que o comportamento como a agressividade e a impulsividade ocasionam isolamento social (SILVA; SOUZA, 2005).

Os indivíduos com TDAH possuem baixa tolerância à espera, dificuldade de inibir comportamentos, alta necessidade de recompensa imediata, falha na previsão das consequências, prejuízo nas habilidades de planejamento e de interrupção das tarefas, déficit de autorregulação e presença de respostas imediatas, porém imprecisas (ASSEF; CAPOVILLA; CAPOVILLA, 2007).

Um estudo realizado no Brasil por Gomes et al. (2007) verificou-se que o conhecimento da população sobre o transtorno do déficit de atenção/ hiperatividade (TDAH) e de médicos, psicólogos e educadores sobre aspectos clínicos do transtorno. Foram avaliados 2.117 indivíduos com idade maior e igual 16 anos, 500 educadores, 405 médicos (128 clínicos gerais, 45 neurologistas, 30 neuropediatras, 72 pediatras, 130 psiquiatras) e 100 psicólogos foram entrevistados pelo Instituto Datafolha. A abordagem foi pessoal. Para os profissionais (amostra aleatória simples), os dados foram coletados por telefone em Belém, Fortaleza, Recife, Salvador, Brasília, Belo Horizonte, Rio de Janeiro, São Paulo, Curitiba e Porto Alegre. Na população, maior que 50\% acreditavam que medicação para TDAH causa dependência, que TDAH resulta de pais ausentes, que esporte é melhor do que drogas como tratamento e que é viável o tratamento psicoterápico sem medicamentos. Dos educadores, maior que 50\% acreditavam que TDAH resulta de pais ausentes, que o tratamento psicoterápico basta e que os esportes substituem os medicamentos. Entre psicólogos, maior que 50\% acreditavam que o tratamento pode ser somente psicoterápico. Dos médicos, maior que $50 \%$ de 
pediatras e neurologistas acreditavam que TDAH resulta de pais ausentes. Todos os grupos relataram crenças não respaldadas cientificamente, que podem contribuir para diagnostico e tratamento inadequados. É urgente capacitar profissionais e estabelecer um programa de informação sobre TDAH para pais e escolas (GOMES et al., 2007).

\section{Quadro Clínico}

Por muito tempo, o TDAH foi entendido como um diagnóstico com poucas implicações na vida dos pacientes. Comumente as crianças eram avaliadas e tratadas por não especialistas, que se baseavam somente nas queixas de hiperatividade e impulsividade para fechar o diagnóstico, referidas pelos pais ou professores, permanecendo o pensamento da remissão da sintomatologia na puberdade. A crença de que se tratava de um transtorno que acomete principalmente meninos com problemas comportamentais até hoje é bastante difundida. Nas ultimas décadas, tanto a experiência clínica quanto as recentes pesquisas em genética, neuroimagem e neuropsicologia têm contribuído para uma importante mudança na forma de entender o TDAH (SOUZA et al., 2007).

São vários os estudos que mostram que mais de 50\% dos pacientes mantém sintomas na vida adulta, com importante comprometimento na vida social, acadêmica, laboral e familiar. Tanto o processo diagnóstico quanto o tratamento do TDAH são complexos, não só pelos vastos sintomas de desatenção e/ou hiperatividade, mas também pela alta frequência de comorbidades psiquiátricas apresentadas pelos pacientes (SOUZA et al., 2007)

Geralmente, o TDAH é detectado quando a criança inicia a vida escolar e, portanto, sua adaptação fica bastante comprometida, pois elas apresentam dificuldade em familiarizar-se como um novo ambiente e as regras que dele fazem parte. Muitas vezes tornam-se impopulares entre os colegas de classe e professores, podendo levar a outros problemas secundários como dificuldade emocional, de relacionamento familiar e baixo desempenho escolar (LIMA, 2011).

A presença de uma criança com TDAH na família altera consideravelmente a sua estrutura, sendo a mãe a mais atingida, pois geralmente o pai que na maioria das vezes é quem mantém a família, está ausente. Os momentos disponíveis da família para o lazer ou outras reuniões sociais, que deveriam ser momentos agradáveis e de descontração, tornam-se de estresse e desgaste emocional em virtude das atitudes incompatíveis do portador com relação a sua idade. Surgindo situações de constrangimento, principalmente pelo desconhecimento de uma situação especial e o fato de não saber lidar com essa condição (CARVALHO et al., 2012).

Ainda que as pessoas com esse transtorno apresentem uma variedade de dificuldades capazes de colocar vários obstáculos em uma adaptação psicológica adequada, também podem desenvolver 
uma série de qualidades e habilidades especiais. Pode-se identificar a energia e o entusiasmo, a espontaneidade e a capacidade para se divertir e a facilidade para expressar afeto. As características de superatividade e impulsividade do TDAH podem, em seu momento, facilitar sucesso ocupacional e profissional na vida adulta. Existe uma quantidade expressiva de pessoas de destaque que apresentam ou apresentaram quando crianças o TDAH. Essas pessoas ocupam posições de lideranças ou recebem reconhecimento por colaborações na medicina, na política, nas leis, na educação, na arte e no esporte. Mesmo que apresentem dificuldades no desenvolvimento essas pessoas são capazes de alcançar êxitos significativos ao longo de suas vidas (BAUERMEISTER, 2009).

As crianças apresentam uma história de vida de sintomas ou pelo menos um período de vários meses de sintomatologia. A presença dos sintomas que iniciam após um desencadeante psicossocial, deve alertar o clínico para a possibilidade de que a desatenção, a hiperatividade ou a impulsividade sejam mais sintomas do que parte de um quadro de TDAH. É importante que a persistência dos sintomas em locais diferentes e ao longo do tempo seja avaliada (ROHDE et al., 2004).

A sintomatologia precisa ocorre em vários ambientes da vida do indivíduo, como a escola e em casa, e se mantém constante ao longo do período de avaliação. Os sintomas que ocorrem apenas em casa ou somente na escola devem alertar o médico para a possibilidade de que a desatenção, a hiperatividade ou a impulsividade possam ser apenas parte de uma situação familiar conturbada ou de um sistema de ensino ineficiente. Flutuação de sintomatologia com períodos assintomáticos não são características do TDAH. Portanto, sintomas isolados ou combinações de dois ou três sintomas, mesmo que frequentes tem pouco valor diagnóstico (ROHDE et al., 2004).

O Transtorno de déficit de atenção e hiperatividade/impulsividade é uma patologia reconhecida pela Organização Mundial de Saúde (OMS). O TDAH permanece como sendo um diagnóstico com forte embasamento científico, diagnosticado em muitos países diferentes culturalmente como o Irã, Estados Unidos, China, Brasil, México. Segundo a Associação Médica Americana (1998) apud Mattos (2013b), uma das mais influentes e respeitadas do mundo afirmou: "O TDAH é um dos transtornos mais bem estudados na medicina e os dados gerais sobre sua validade são muito mais convincentes que a maioria dos transtornos mentais e até mesmo que muitas condições médicas" (MATTOS, 2013b, p. 18).

A tríade sintomatológica clássica da síndrome caracteriza-se por desatenção, hiperatividade e impulsividade. Independente do sistema de classificação utilizado, as crianças com TDAH são facilmente reconhecidas em clínicas, em escolas e em casa. A desatenção pode ser identificada pelos seguintes sintomas: dificuldade de prestar atenção a detalhes ou errar por descuido em atividades escolares e de trabalho; dificuldade para manter a atenção em tarefas ou atividades lúdicas; parecer não escutar quando lhe dirigem a palavra; não seguir instruções e não terminar tarefas escolares, 
domésticas ou deveres profissionais; dificuldade em organizar tarefas e atividades; evita-se envolver em tarefas que exijam esforço mental constante; perde coisas necessárias para tarefas ou atividades; facilmente se distraem por estímulos alheios as tarefas e apresentam esquecimento em atividades diárias (ROHDE et al., 2000).

A hiperatividade se caracteriza pela presença freqüente dos seguintes sintomas: agitar as mãos ou os pés ou se mexem na cadeira; abandonar sua cadeira em sala de aula ou outras situações nas quais se espera que permaneça sentado; correr ou escalar em demasia, em situações nas quais isto é inapropriado; pela dificuldade em brincar ou envolver-se silenciosamente em atividade de lazer; estar frequentemente "a mil por hora" ou muitas vezes agir como se estivesse "a todo vapor"; e falar em demasia. Os sintomas de impulsividade são: dar respostas precipitadas antes das perguntas terem sido concluídas; com frequência ter dificuldades em esperar a sua vez; e interromper assuntos de outras pessoas (ROHDE et al., 2000).

Os pacientes portadores de TDAH apresentam alterações especificas em uma função cognitiva chamada de Função Executiva (FE). Esta é a função mental que coordena a memória imediata, memória imediata verbal, autorregulação dos afetos e permite a reconstituição e análise do próprio comportamento. Alterações dessa função podem acarretar um menor controle dos impulsos, dificuldades de reter informações, respostas verbais inadequadas e problemas no controle motor a estímulos. Alguns autores consideram a FE o "maestro da sinfonia mental do indivíduo". Todas as pessoas, com ou sem TDAH, podem apresentar algum grau de alteração da FE. Entretanto, o TDAH pode ser entendido como o extremo de um comportamento e de um mau funcionamento da FE, uma vez que esta população apresenta um grande número de problemas na área dos processos de ensino e aprendizado, o que acarreta dificuldades na aprendizagem de novos conteúdos (GREVET; ABREU; SHANSIS, 2003).

As funções executivas podem ser divididas entre quatro subconjuntos: (1) volição; (2) planejamento; (3) ação intencional; e (4) desempenho efetivo (LEZAK et al., 2004 apud SABOYA et al., 2007). A volição é a capacidade de estabelecer objetivos. É necessária a motivação e consciência de si e do ambiente. O planejamento é a capacidade de organizar e prever ações para atingir um objetivo. A habilidade de planejar requer capacidade para tomar decisões, desenvolver estratégias, estabelecer prioridades e controlar impulsos. A ação intencional é a efetivação de um objetivo e planejamento, gerando uma ação produtiva. O desempenho efetivo é a capacidade de auto monitorar, auto dirigir e auto regular a intensidade, o ritmo e outros aspectos qualitativos do comportamento e da ação, ou seja, é um controle funcional (GONÇALVES et al., 2013; SABOYA et al., 2007).

Segundo LOPES et al. (2005) e SABOYA et al. (2007), a constelação da síndrome disexecutiva no TDAH pode apresentar uma variedade de manifestações. Entre elas, ocorre: 
a) procrastinação, ou seja, o indivíduo tende a postergar tarefas, principalmente quando envolvem maior necessidade de atenção ou levam a uma recompensa a longo prazo;

b) alternância de tarefas, deixando-as incompletas, em função de uma baixa capacidade de persistir em uma tarefa;

c) labilidade motivacional, apresentando interesse fugaz com necessidade de buscar novidades;

d) dificuldade de focalização e sustentação da atenção, revelando maior sensibilidade à distração, com dificuldade para filtrar estímulos internos e externos. Necessita de lembretes para manter uma tarefa habitual, apresentando inconstância e abandono precoce de tarefas;

e) dificuldade de organização e hierarquização, apresentando problemas para estabelecer prioridades e distinguir importâncias;

f) menor velocidade de processamento;

g) manejo deficiente da frustração e da modulação do afeto, apresentando baixa tolerância e limiar para frustração com baixa auto-estima, hipersensibilidade a críticas e irritabilidade;

h) deficiência de memória de trabalho com dificuldade de manipular informações verbais e não-verbais em curto espaço de tempo, seguimento de seqüências;

i) deficiência de memória prospectiva, gerando esquecimentos de responsabilidades e objetivos estipulados.

A qualidade de vida dessas crianças é diminuída. Os elogios desaparecem e frequentemente a mentira toma conta da situação, por que elas não querem mais ser as erradas. Ao perceberem as queixas da escola e dos pais, envergonham-se de suas atitudes, mas não conseguem mudar a situação sem a ajuda de profissionais preparados. Um dos grandes vilões da criança com TDAH sem tratamento adequado é a autoestima. Dificuldades de relacionamento com a família, a escola e os amigos são comuns e prejudicam a qualidade de vida dessas crianças. Muitas delas chegam a adolescência e aprendem a driblar intuitivamente os sintomas, mas eles existem e podem atrapalhar o seu desenvolvimento na idade adulta (FRIAÇA, 2010; MATTOS; COUTINHO, 2007; MATTOS et al., 2012). 


\section{Diagnóstico}

O diagnóstico do TDAH é fundamentalmente clínico, baseado em critérios operacionais claros e bem definidos proveniente do sistema classificatório como DSM IV, em ANEXO A, ou a CID- 10, em ANEXO B. O Manual de diagnóstico e estatística das doenças mentais IV (DSM-IV) propõe a necessidade de pelo menos seis sintomas de desatenção e/ou seis sintomas de hiperatividade/impulsividade e divide o TDAH em três tipos: a)com predomínio de sintomas de desatenção; b) com predomínio de hiperatividade/impulsividade; c) combinado.

O tipo com predomínio de desatenção é mais frequente no sexo feminino, e parece apresentar conjuntamente com o tipo combinado uma taxa mais elevada do prejuízo acadêmico. As crianças com características de hiperatividade e impulsividade são mais agressivas do que as que possuem os outros dois tipos (ROHDE et al., 2000).

O DSM IV e o CID-10 relatam que o início dos sintomas causadores de prejuízo ocorre antes dos sete anos de idade, para o diagnóstico do transtorno. Entretanto esse critério é derivado somente da opinião de um comitê de "experts" no TDAH, sem nenhuma evidencia cientifica que sustente sua validade clínica (BARKLEY; BIEDERMAN, 1997 apud ROHDE et al., 2004).

Indivíduos que apresentam uma maior vulnerabilidade ao transtorno do que a população em geral, mas sem que ela chegue a ser acentuada, podem apresentar sintomas significativos a partir do momento que a exigência ambiental passa ser bem maior. Em crianças isso pode ocorrer a partir de uma terceira ou quarta série do ensino fundamental, onde as necessidades de funções de planejamento, execuções, organizações e atenção são mais exigidas para a realização das tarefas escolares. Dessa forma, o médico não deve descartar a possibilidade do diagnóstico em pacientes que apresentem sintomas causadores de prejuízo após os sete anos (ROHDE et al., 2004).

Utilizando o DSM IV como instrumento diagnóstico, encontramos uma maior prevalência do que se for usada o CID-10, sendo que os pacientes identificados como portadores do Transtorno Hipercinético (TH) são muito semelhantes aos portadores do TDAH do tipo combinado. O subtipo predominantemente hiperativo/impulsivo e predominantemente desatento não se enquadram na classificação da CID-10, e seu subdiagnósticos causa impacto considerável em termos epidemiológicos e prognósticos, envolvendo também alterações na resposta terapêutica (LOUZÃ NETO, 2010).

Sendo assim, na proposta para a formulação do DSM V e CID-11, espera-se uma maior uniformidade de critérios diagnósticos, de forma a contemplar todos os indivíduos acometidos pelo transtorno para que possam receber a atenção adequada e o tratamento correto. No mérito dessa questão, vale a pena ressaltar que a imposição de uma idade de início para o diagnóstico do transtorno é arbitrária e não apresenta valor significativo, já que estudos comprovam que não há nenhuma 
diferença significativa em taxas de comorbidades e prejuízo funcional nos pacientes com surgimento dos sintomas antes e depois dos 6 e 7 anos, de acordo com o CID-10 e o DSM IV (ROHDE, 2008 apud ASBAHR; COSTA; MORIKAWA, 2010).

O DSM teve a sua quinta edição lançada no congresso de psiquiatria, ocorrido em São Francisco, em maio de 2013. O seu planejamento começou muitos anos antes, em 1999, quando uma série de colaborações delineou as questões que precisavam ser mais bem esclarecidas na quarta edição, DSM-IV, sempre através de pesquisas científicas. Uma segunda fase, entre 2003 e 2008, compreendeu 13 conferências internacionais com os maiores especialistas de cada uma das diferentes áreas (transtornos infantis, transtornos de ansiedade, dependência de drogas, doenças degenerativas, etc.), incluindo o TDAH. Parte do trabalho realizado por todos os pesquisadores será utilizada na confecção do CID-11, a futura versão da Classificação Internacional de Doenças proposta pela Organização Mundial da Saúde (OMS), que é a referencia oficial para diagnósticos na maioria dos países do mundo, incluindo o Brasil (MATTOS, 2013a).

Alguns diagnósticos psiquiátricos pouco ou nada mudaram na quinta edição, outros se modificaram de modo significativo; alguns diagnósticos novos foram propostos e outros foram abandonados. No caso do TDAH, foram poucas as modificações. A lista de 18 sintomas, sendo 9 de desatenção, 6 de hiperatividade e 3 de impulsividade (estes dois últimos computados conjuntamente) permaneceu a mesma que na edição anterior. O ponto-de-corte para o diagnóstico, isto é, o número de sintomas acima do qual se faz o diagnóstico, também permaneceu o mesmo (6 sintomas de desatenção e/ou 6 sintomas de hiperatividade-impulsividade). No caso de adultos, este número passou para 5 sintomas, o que é um novo critério (MATTOS, 2013a).

A lista de sintomas de desatenção e hiperatividade-impulsividade compreende o critério A. Todos estes sintomas, para serem considerados clinicamente significativos, devem estar presentes pelo menos durante 6 meses e serem incoerentes com a idade do indivíduo. A necessidade de haver comprometimento de pelo menos duas áreas diferentes, que correspondem ao critério $\mathrm{C}$, permaneceu como antes. A necessidade de haver claro comprometimento na vida acadêmica, social, profissional, que faz parte do critério $\mathrm{D}$, também permaneceu idêntica $\mathrm{O}$ critério $\mathrm{E}$ foi modificado em relação à DSM-IV. Antes, não era possível fazer o diagnóstico de TDAH caso houvesse um quadro de autismo, o que agora é possível. Entretanto, permanecem as exigências de que os sintomas não ocorrerem exclusivamente durante outro quadro como na esquizofrenia e não serem mais bem explicados por outro transtorno como na ansiedade e depressão (MATTOS, 2013a).

$\mathrm{O}$ critério $\mathrm{B}$, que determina a idade de início dos sintomas, também se modificou. Anteriormente, era necessário demonstrar que os sintomas estivessem presentes antes dos 7 anos de idade, o que era particularmente difícil no caso de adultos com TDAH que geralmente tem dificuldade 
para lembrar-se deste período e cujos pais já são mais velhos. O limite de idade foi modificado para 12 anos, algo que alguns grupos de pesquisa já vinham fazendo anteriormente (MATTOS, 2013a).

Os subtipos foram retirados do manual; ao invés disso, optou-se pelo emprego do termo apresentação, denotando que o perfil de sintomas atuais pode se modificar com o tempo, $\mathrm{O}$ termo subtipo favorecia uma interpretação errada que aquela era uma subcategoria estável, fixa, do TDAH. As apresentações mantêm as mesmas divisões que os antigos subtipos: com predomínio de desatenção, com predomínio de hiperatividade-impulsividade e apresentação combinada (MATTOS, 2013a).

O DSM-V traz a opção de TDAH com remissão parcial, que deve ser empregado naqueles casos onde houve diagnóstico pleno de TDAH anteriormente, porém com um menor numero de sintomas atuais. Uma última novidade desta quinta edição é a possibilidade de se classificar o TDAH em leve, moderado e grave, de acordo com o grau de comprometimento que os sintomas causam na vida do indivíduo (MATTOS, 2013a). Diferenças entre critérios para TDAH (DSM IV) e TH (CID10), segundo Biederman e Faraone (2005) apud Asbahr, Costa e Morikawa (2010).

\section{* Diagnóstico de TDAH segundo o DSM IV:}

- Sintomas

Um ou ambos dos seguintes: a) Pelo menos 6 (de 9) sintomas de desatenção; b) Pelo menos 6 (de 9) sintomas de hiperatividade ou impulsividade.

- Particularidade

a) Algum prejuízo decorrente dos sintomas está presente em mais de um contexto

\section{* Diagnóstico de TH segundo o CID -10:}

- Sintomas

$\checkmark$ Todos os que seguem:

a) Pelo menos 6 (de 8) sintomas de desatenção;

b) Pelo menos 3 (de 5) sintomas de hiperatividade;

c) Pelo menos 1 (de 4) sintomas de impulsividade.

- Particularidade

Os critérios são preenchidos em mais de uma situação. 
$\mathrm{Na}$ forma predominantemente hiperativa/impulsiva ou combinada as crianças tendem a incomodar mais em sala de aula e criar um ambiente mais confuso e desorganizado, sendo estas mais facilmente encaminhadas para avaliação médicas, a pedido dos próprios educadores. As crianças que possuem a forma desatenta são comumente diagnosticadas mais tardiamente, porque a hiperatividade não é tão importante, mas sim, o aluno não perturbar o ambiente. A criança desatenta tem um rendimento menor que os demais alunos, apenas quando o conteúdo se torna maior e mais complexo é que a desatenção se torna mais evidente (MATTOS, 2013b).

Segundo Mattos (2013b) uma lista de problemas que ocorre com maior freqüência em portadores de TDAH, quando comparado a outras pessoas da mesma idade e classe social foi demonstrado em estudos científicos recente. Os problemas foram correlacionados com a faixa etária:

* Em crianças e adolescentes ocorre com maior frequência:

Acidentes; Problemas de aprendizado escolar; Reprovações; Expulsões; Abandono escolar; Incidência de abuso de álcool e drogas ao final da adolescência; Depressão;Ansiedade.

Em adultos observou-se que há maior incidência:

Desemprego; Divórcio; Menos anos de escolaridade completados; Acidentes com veículos; Abuso e álcool e drogas; Depressão; Ansiedade.

Os psiquiatras afirmam que para diferenciar um paciente com TDAH do que não apresenta o transtorno, são feitas comparações. Suas execuções produtivas, adaptação social às exigências do ambiente e sua capacidade de autocontrole são confrontadas. A suspeita em torno do diagnóstico do TDAH é marcada pelo dilema metodológico da objetividade científica. Embora os exames de imagem tentem mostrar o contrário, a linha que separa o indivíduo TDAH do sujeito normal é tão tênue, que na clínica e na vida prática ela não pode ser traçada e nem visualizada. Apesar dos avanços dos métodos de visualização cerebral, na prática diagnóstica eles não possuem muita relevância. Até o momento, nenhum exame específico ou teste foi exato para a identificação do TDAH. O seu diagnóstico continua sendo realizado através de um método misto, que inclui testes psicológicos, história clínica, entrevistas com pais e professores e avaliação do desempenho escolar (CALIMAN, 2008). 


\section{Conclusão}

Apesar de ser citada na literatura médica há pelo menos um século, o TDAH ainda é alvo de controvérsias. É um transtorno desconhecido por muitas pessoas e questionado sobre sua validade como diagnóstico médico e bastante confundido com características de cada individuo. É frequentemente subdiagnosticado e subtratado em nosso meio.

Durante muito tempo acreditava-se que a patologia era restrita somente à infância, mas hoje se sabe que pode persistir até a vida adulta. Devido ao seu início precoce e a suas características crônicas, prejudica o desenvolvimento e o convívio social, podendo trazer sérias limitações que futuramente serão difíceis de ser superadas. Em cada etapa de vida do paciente o TDAH é sentido diretamente por seu portador e por pessoas que convivem frequentemente com ele.

$\mathrm{Na}$ infância, o TDAH em geral se associa a dificuldades na escola e no relacionamento com demais crianças, pais e professores. As crianças são tachadas como "avoadas", "vivem no mundo da lua" e geralmente "estabanadas" ou "ligado por um motor", isto é, não param quietas por muito tempo.

O transtorno afeta todos aqueles que convivem com o portador da doença. Por isso, encarála é o primeiro passo para o seu controle. Professores, psicólogos e médicos trabalham em parceria com a família para apoiar a criança ou o adulto em seu dia a dia, diminuindo os impactos que o transtorno pode acarretar em seu aprendizado e desenvolvimento social. O tratamento adequado, aliado à terapia medicamentosa, melhora a vida dos pacientes.

O transtorno é visto pela família como um fator que dificulta o convívio e o dia a dia. Tendo em vista o caráter bidirecional das relações familiares, pode-se dizer que não é somente a criança que influencia o comportamento dos pais, mas o contrário também ocorre. Levando em consideração essa recíproca nas relações, o tratamento do TDAH exige, além da redução dos sintomas, a inclusão dos pais na terapia da criança, com o intuito de analisar as interações familiares, modificar interações e atribuições disfuncionais e aumentar o número de interações positivas.

O papel do professor é fundamental para auxiliar médicos e psicopedagogos no diagnóstico e no tratamento do TDAH, visto que a hiperatividade, a desatenção e a impulsividade, ficam evidentes no período escolar, quando é preciso aumentar o nível de concentração para melhorar o aprendizado. Dessa forma, é importante o professor estar bem informado e ter conhecimentos acerca do assunto, a fim de identificar, compreender, acolher e distinguir as crianças que realmente possuem a patologia.

$\mathrm{O}$ portador do TDAH precisa ter na escola um acompanhamento e um ambiente adequado para seu aprendizado, já que não possui componentes para inibir fatores distratores, refletindo em tumulto na sala de aula, na vida dos colegas e dos seus professores. É preciso aplicar uma ação didática-pedagógica direcionada para estas crianças, visando elevar sua auto-estima, valorizar 
atividades diversificadas e criativas, com estimulação do lúdico, tentando usar essas estratégicas como forma de aprendizagem.

O profissional médico deve diagnosticar e tratar corretamente crianças e adultos com TDAH, de acordo com diretrizes estabelecidas pela comunidade científica, deve fornecer ao portador e a seus familiares informações científicas e atualizadas acerca da natureza do TDAH, bem como suas consequências e as formas disponíveis de tratamento. O médico deve oferecer um tratamento sempre individualizado, levando em consideração aspectos específicos do portador, sua família e o contexto sócio-cultural em que vivem, tentando integrá-lo como co-participante da terapêutica, minimizando o sofrimento.

\section{Referências}

ABREU, N.; OLIVEIRA, I. R. IN: LOUZA NETO, M. R. TDAH: transtorno de déficit de atenção hiperatividade ao longo da vida. Porto Alegre: Artmed, 2010, p.22 - 37.

ASBAHR, F. R.;COSTA, C. Z. G.; MORIKAWA, M. Criança e adolescente. In: LOUZÃ NETOP, M. R. et al. TDAH: transtorno de déficit de atenção, hiperatividade ao longo da vida. Porto Alegre: Artmed, 2009, p. $152-159$.

ASSEF, Ellen Carolina dos Santos; CAPOVILLA, Alessandra Gotuzo Seabra; CAPOVILLA, Fernando César. Avaliação do controle inibitório em TDAH por meio do teste de geração semântica. Psicologia: Teoria e Pratica, v. 9, n. 1, 2007.

BAUERMEISTER, José. Hiperativo impulsivo distraído: você conhece?. São Paulo: Elevação, 2009.

BARKLEY, R. A. Histórico. In Transtorno de déficit de atenção e hiperatividade: manual para diagnóstico e tratamento.3. ed. Porto Alegre: Artmed, 2008, p.15 - 87.

BELLÉ, A. et al. Estresse e adaptação psicossocial em mães de crianças com transtorno de déficit de atenção/hiperatividade. Psicologia: Reflexão e Crítica, v. 22, n. 3, p. 317-325, 2009.

CALIMAN, Luciana Vieira. O TDAH: entre as funções, disfunções e otimização da atenção. Psicologia em Estudo, Maringá, v. 13, n. 3, p. 559-566, jul./set. 2008.

CALIMAN, Luciana Vieira. A constituição sócio-médica do "fato TDAH". Psicologia e Sociedade, v. 21, n.1, p. 135-144, 2009.

CARVALHO, J. A. et al. TDAH: Considerações sobre o transtorno do déficit de atenção e hiperatividade. Revista Científica do ITPAC, Araguaína, v. 5, n. 3, 2012.

DIAS, G. et al. Diagnosticando o TDAH em adultos na prática clínica. Jornal Brasileiro de Psiquiatria, v. 56, n.1, p. 9 - 13, 2007. 
COMAN, T.; CONTINI, V. BAU, C. H. D. Genética. In: LOUZA NETO, M. R. TDAH: transtorno de déficit de atenção hiperatividade: ao longo da vida. Porto Alegre: Artmed, 2010, p. 40 - 57.

FONTANA, Rosiane da Silva et al. Prevalência de TDAH em quatro escolas públicas brasileiras. Arquivo de Neuropsiquiatria, v. 65, n. 1, p. 134-137, 2007.

FIANÇA, M. G. F. Qualidade de vida. In: LOUZA NETO, M. R. TDAH: transtorno de déficit de atenção hiperatividade ao longo da vida. Porto Alegre: Artmed, 2010, p. 265 - 272.

GATTÁS, I. G. et al. Criança e adolescente. In: LOUZA NETO, M. R. TDAH: transtorno de déficit de atenção hiperatividade ao longo da vida. Porto Alegre: Artmed, 2010, p. 275 - 289.

GOMES, Marcelo et al. Conhecimento sobre o transtorno do déficit de atenção/hiperatividade no Brasil. Jornal Brasileiro de Psiquiatria, v. 56, n. 2, p. 94-101, 2007.

GONÇALVES, Hosana Alves et al. Componentes atencionais e de funções executivas em meninos com TDAH: dados de uma bateria neuropsicológica flexível. Jornal Brasileiro de Psiquiatria, v. 62, n. 1, p. 13-21, 2013.

GREVET, Eugenio Horácio; ABREU, Paulo Belmonte de; SHANSIS, Flávio. Proposta de uma abordagem psicoeducacional em grupos para pacientes adultos com transtorno de déficit de atenção/hiperatividade. Revista de Psiquiatria do Rio Grande do Sul, v. 25, n. 3, p. 446-452, 2003.

GUILHERME, Priscilla Rodrigues et al. Conflitos conjugais e familiares e presença de transtorno de déficit de atenção e hiperatividade (TDAH) na prole: revisão sistemática. Jornal Brasileiro de Psiquiatria, v. 56, n. 3, p. 201-207, 2007.

HECHTMAN, L. Altention - déficit: hyperactivity disorder. In: KAPLAN, Harold I.; SADOCK, Benjamin J. Kaplan e Sadock's comprehensive textbook of psychiatry. 8. ed. Philadelphia: Lippincott Williams, 2005, p. 3183-3204.

ITABORAHY, Claudia; ORTEGA, Francisco. O metilfenidato no Brasil: uma década de publicações. Ciência e Saúde Coletiva, v.18, n. 3, p. 803 - 816, 2013.

LAKATOS, E. M.; MARCONI, M. A. Metodologia do trabalho científico. São Paulo: Atlas, 1991.

LIMA, F. A. O. Transtornos do déficit de atenção e hiperatividade: entendendo melhor a criança com TDAH no contexto da escola pública. 2011. Monografia (Especialização) - Universidade de Brasília, Brasília, 2011.

LOPES, Regina Maria Fernandes; NASCIMENTO, Roberta Fernandes Lopes do; BANDEIRA, Denise Ruschel. Avaliação do transtorno de déficit de atenção/hiperatividade em adultos (TDAH): uma revisão de literatura. Avaliação Psicológica, v. 4, n. 1, p. 65-74, 2005.

LOUTFI, Karina Soares and CARVALHO, Alysson Massote. Possíveis interfaces entre TDAH e epilepsia. Jornal Brasileiro de Psiquiatria, v. 59, n.2, p. 146-155, 2010.

LOUZÃ, Mario R.; MATTOS, Paulo. Questões atuais no tratamento farmacológico do TDAH em adultos com metilfenidato. Jornal Brasileiro de Psiquiatria, v. 56, n. 1, p. 53-56, 2007.

LOUZÃ NETO, M. R. TDAH: transtorno de déficit de atenção hiperatividade ao longo da vida. Porto Alegre: Artmed, 2010. 
LOBATO, R. S. Educação física adaptada para crianças com déficit de atenção e hiperatividade. 2011. Monografia (Especialização em Desenvolvimento Humano) - Universidade de Brasília, Brasília, 2011.

LUNA, S. V. Planejamento de pesquisa: uma introdução. São Paulo: EDUC, 1997.

MARIANO, B. S. O exercício físico e o transtorno de déficit de atenção: um estudo de revisão. 2011. Monografia (Trabalhos de Conclusão de Curso) - Universidade do Estado de Santa Catarina, Florianópolis, 2011.

MATTOS, P. Adulto. In: LOUZA NETO, M. R. TDAH: transtorno de déficit de atenção hiperatividade ao longo da vida. Porto Alegre: Artmed, 2010, p. 161 - 169.

. Entenda o TDAH nos critérios do DSM - V. In: ABDA, 2013a. Disponível em: < http://www.tdah.org.br/br/textos/textos/item/964-entenda-o-tdah-nos-crit\%C3\%A9rios-do-dsmv.html>. Acesso em: jan. de 2014.

No mundo da lua: transtornos de déficit de atenção com hiperatividade, TDAH. 13 ed. [S. L]: Associação Brasileira de Déficit de Atenção, 2013b.

MATTOS, Paulo et al. A Multicenter, Open-Label Trial to Evaluate the Quality of Life in Adults With ADHD Treated With Long-Acting Methylphenidate (OROS MPH) Concerta Quality of Life (CONQoL) Study. Journal of Attention Disorders, v. 17, n. 5, p. 444-448, 2012.

. Adaptação transcultural para o português da escola Adult- Report Scale para avaliação do transtorno de déficit de atenção hiperatividade (TDAH) em adultos. Revista de Psiquiatria Clínica., v. 33 , n. 4 , p. $188-194,2006 a$.

Painel brasileiro de especialistas sobre diagnóstico do transtorno de déficit de atenção/hiperatividade (TDAH) em adultos. Revista de Psiquiatria., v. 28, n. 1, p. 50-60, 2006 b.

Transtornos alimentares comórbidos em uma amostra clínica de adultos com transtorno do déficit de atenção com hiperatividade. Revista Brasileira de Psiquiatria, v. 26, n. 4, p. 248-50, 2004.

MATTOS, Paulo; COUTINHO, Gabriel. Qualidade de vida e TDAH. Jornal Brasileiro de Psiquiatria, v. 56, n.1, p. 50-2, 2007.

MORAES, César de; SILVA, F. M. B. N.; ANDRADE, E. R. Diagnóstico e tratamento de transtorno bipolar e TDAH na infância: desafios na prática clínica. Jornal Brasileiro de Psiquiatria, v. 56, n. 1, p. 19-24, 2007.

NAGEL, Bonnie J. et al. Altered white matter microstructure in children with attentiondeficit/hyperactivity disorder. Journal of the American Academy of Child \& Adolescent Psychiatry, v. 50, n. 3, p. 283-292, 2011.

ORTEGA, Francisco et al. A ritalina no Brasil: produções, discursos e práticas. InterfaceComunicação, Saúde, Educação, v. 14, n. 34, p. 499 - 510, jul./set. 2010.

OSWALD, S. H.; KAPPLER, C. O. Relações familiares de criança com TDAH. In: LOUZÃ NETO, M. R. TDAH: transtorno de déficit de atenção hiperatividade ao longo da vida. Porto Alegre: Artmed, 2010 , p. $368-375$. 
PASSO, R. B. F.; LÓPEZ, J. R. R. A. Sindrome de Gilles de la Tourette associada ao transtorno de déficit de atenção com hiperatividade: respostas clínicas satisfatória a inibidor da recaptura de serotonina e metilfenidato. Jornal Brasileiro de Psiquiatria, v. 59, n. 2, p. 160 - 162, 2010

PEREIRA, H. S.; ARAUJO, A. P. Q. C.; MATTOS, P. Transtorno do déficit de atenção e hiperatividade (TDAH): aspectos relacionados á comorbidade com distúrbios da atividade motora. Revista Brasileira de Saúde Materno Infantil, Recife, v. 5, n.4, p.391 - 402, out./dez. 2005.

POSSA, M de Aguiar; SPANEMBERG, L.; GUARDIOLA, A.

Comorbidades do transtorno de déficit de atenção e hiperatividade em crianças escolares. Arquivos de Neuropsiquiatria, v. 63, n. 2, p. $479-483,2005$.

REGALLA, Maria Angélica; GUILHERME, Priscilla Rodrigues; SERRA-PINHEIRO, Maria Antônia. Resiliência e transtorno do déficit de atenção/hiperatividade. Jornal Brasileiro de Psiquiatria., v. 56, n. 1, p. 45-49, 2007.

REINHARDT, Marcelo C.; REINHARDT, Caciane AU. Attention deficit-hyperactivity disorder, comorbidities, and risk situations. Jornal de Pediatria, v. 89, n. 2, p. 124-130, 2013.

RICHTER, B. R. O professor atento ao TDAH: a hiperatividade e indisciplina na revista nova escola. In: ANPED SUL SEMINÁRIO DE PESQUISA EM EDUCAÇÃO DA REGIÃO SUL, 9., 2012. [Santa Catarina]. Anais... [Santa Catarina]: ANPED SUL, 2012, p. 2- 13.

ROHDE, L. A. E. et al. Transtorno de déficit de atenção: hiperatividade. Revista Brasileira de Psiquiatria, v. 22, p. 7 - 11, 2000.

ROHDE, Luis Augusto et al. Transtorno de déficit de atenção/hiperatividade na infância e na adolescência: considerações clínicas e terapêuticas. Revista de Psiquiatria Clínica, v. 31, n. 3, p. 124-131, 2004.

ROHDE, R. A.; HALPERN, R. Transtorno de déficit de atenção hiperatividade: atualização. Jornal de Pediatria, v. 80, n. 2, 2004.

ROMAN, Tatiana; ROHDE, Luis Augusto; HUTZ, Mara Helena. Genes de suscetibilidade no transtorno de déficit de atenção e hiperatividade. Revista Brasileira de Psiquiatria, v. 24, n. 4, p. 196-201, 2002.

ROMAN,Tatiana;CONTINI,Verônica;BAU,C.H.D.Genética.In:LOUZÃ NETO, M. R. TDAH: transtorno de déficit de atenção hiperatividade ao longo da vida. Porto Alegre: Artmed, 2010, p. 40 60 .

ROMANO, M. O transtorno do déficit de atenção hiperatividade $\mathrm{x}$ dependência de álcool e outras substancias. In: RAMOS, S. P. Comorbidades: transtornos mentais $\mathrm{x}$ transtornos por uso de substancias de abuso. [s. 1: s. n]. [19- -]. p. $12-15$.

SABOYA, Eloisa et al. Disfunção executiva como uma medida de funcionalidade em adultos com TDAH. Jornal Brasileiro de Psiquiatria, v. 56, n. 1, 2007.

SCHMITZ, Marcelo; POLANCZYK, Guilherme; ROHDE, Luis Augusto Paim. TDAH: remissão na adolescência e preditores de persistência em adultos. Jornal Brasileiro de Psiquiatria, v. 56, n. 1, p. 25-9, 2007. 
SENO, M. P. Transtornos de déficit de atenção e hiperatividade (TDAH): o que os educadores sabem?. Revista de Psicopedagogia, v. 27, n. 84, p. $334-43,2010$.

SERRA-PINHEIRO, Maria Antonia; SCHMITZ, Marcelo; MATTOS, Paulo. Transtorno desafiador de oposição: uma revisão de correlatos neurobiológicos e ambientais, comorbidades, tratamento e prognóstico. Revista Brasileira de Psiquiatria, v. 26, n. 4, p. 273-276, 2004.

SILVA, E. L.; MENEZES, E. M. Metodologia da Pesquisa e Elaboração de Dissertação. 3. ed. Florianópolis: [s.n.], 2000.

SILVA, M. P. Neuroimagem. In: LOUZÃ NETO, M. R. TDAH: transtorno de déficit de atenção hiperatividade ao longo da vida. Porto Alegre: Artmed, 2010, p. 82 - 96.

SILVA, Rejane Augusta; SOUZA, Luiz Augusto de Paula. Aspectos lingüísticos e sociais relacionados ao transtorno de déficit de atenção/hiperatividade. Revista CEFAC, v. 7, n. 3, p. 295299, 2005.

SOUZA, Isabella GS et al. Dificuldades no diagnóstico de TDAH em crianças. Jornal Brasileiro de Psiquiatria, v. 56, p. 14-18, 2007.

SZOBOT, Claudia M. et al. Neuroimagem no transtorno de déficit de atenção/hiperatividade. Revista Brasileira de Psiquiatria, v. 23, p. 32-35, 2001.

SZOBOT, Claudia M.; ROMANO, Marcos. Co-ocorrência entre transtorno de déficit de atenção/hiperatividade e uso de substâncias psicoativas. Jornal Brasileiro de Psiquiatria, v. 56, n. 1, p. 39-44, 2007.

VIDAL-ESTRADA, R. et al. Psychological treatment of attention defi cit hyperactivity disorder in adults: a systematic review. Actas Españolas de Psiquiatría, v. 40, n. 3, p. 147-54, 2012.

\section{Como citar este artigo (Formato ISO):}

BEZERRA, Catarina F.M.B., TELLES, Maria Valéria L. BEZERRA, Maria Iaponira F.; RIBEIRO, Samuel O.; SÁ, Noyla D.A. O Transtorno do Défict de Atenção e Hiperatividade. Id on Line Revista de Psicologia, Julho de 2014, vol.8, n.23, p. 221-242. ISSN 1981-1179. 